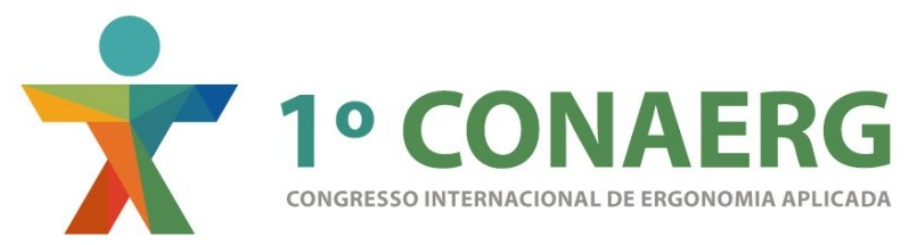

\title{
PERFIL SOCIOECONÔMICO E SAÚDE DOS TRABALHADORES EM INDÚSTRIAS DA REGIÃO AMAZÔNIA
}

\author{
Rodrigo Canto Moreira (1); \\ Brenda Rassy Carneiro Maradei (2) \\ (1) Universidade Federal do Pará, Mestrando \\ e-mail: rodrigocanto.fisio@hotmail.com \\ (2) Universidade Federal do Pará, Mestre \\ e-mail: brendacarneiro@yahoo.com.br
}

\begin{abstract}
RESUMO
Este trabalho visa apresentar o perfil socioeconômico dos trabalhadores industriais paraenses e dados relativos à saúde, identificando alguns dos problemas que merecem atenção do empresariado e das autoridades governamentais. Para tanto, foi realizado um estudo descritivo e transversal com análises secundárias de dados, envolvendo 9041 trabalhadores de 56 indústrias nacionais presentes na região amazônica brasileira. Os resultados indicam um menor número de trabalhadores que relatam diabetes e hipertensão arterial sistêmica e maior incidência de problemas de coluna, quando comparados à população brasileira e indicativos de depressão. Conclui-se que são necessárias maiores políticas públicas e privadas, relativas à vigilância em saúde.
\end{abstract}

Palavras- chave: Saúde do trabalhador, Vigilância em saúde, Ergonomia industrial, Psicopatologia do trabalho.

\begin{abstract}
This article aims to present the socioeconomic profile of Paraense industrial workers and health data, identifying some problems that need attention from entrepreneurs and government authorities. Therefore, It was conducted a descriptive and cross-sectional study with secondary data analysis, involving 9,041 workers from 56 national industries of the Brazilian Amazon region. The results demonstrate a smaller number of workers who reported diabetes and hypertension and higher incidence of spinal disorders, when compared to the Brazilian population and, some indicative of depression. We conclude that it is necessary more public and private policies regarding to health surveillance.
\end{abstract}

Key words: Occupational health, Health Surveillance, Industrial Ergonomics, Work Psychopathology.

\section{INTRODUÇÃO}

De tempos em tempos, o mundo do trabalho vem passando por transformações intensas, sendo que nas últimas décadas, com o advento de novas tecnologias, essas mudanças 
estão tendo reflexo, tanto na produção quanto na cultura organizacional, de forma bem peculiar; já que com o avanço gradual na organização da produção, com o processo de globalização e a nova ordem estabelecida nas relações entre capital e trabalho, as relações conflituosas no trabalho ainda estão presentes, originando novos processos de adoecimento e dificuldades para retorno e, sobretudo, permanência no trabalho (TOLDRA et. al., 2010).

Os efeitos da nova configuração do trabalho (informatização e robotização na oferta de produtos e serviços) aumentam a necessidade de um maior conhecimento a respeito das consequências provocadas por mudanças recentes, tendo em vista que o aumento do controle sobre prazos e metas, os quais estão bem delineados para as organizações, podem gerar agravos à saúde do trabalhador e que antes não existiam. A rotina está mais intensa dentro das organizações capitalistas devido à busca pelo lucro que está cada vez mais acirrada, podendo até mesmo atropelar a valorização do funcionário (Couto, 1995).

Conhecer a saúde do trabalhador favorece a manutenção de práticas motivacionais, que beneficiam o trabalhador em sua saúde e a empresa em sua produtividade/lucratividade. Estudos sobre a promoção de saúde no local de trabalho têm quantificado a relação entre boa saúde e aumento de produtividade (SANTOS \& VAJDA, 2007). Além disso, a Política Nacional de Segurança e de Saúde do Trabalhador (BRASIL, 2011) e a Política Nacional da Saúde do Trabalhador e da Trabalhadora (BRASIL, 2012) asseguram ao trabalhador o suporte necessário à melhoria e à efetivação dos serviços direcionados a eles pelas empresas privadas e pelo governo.

Estatísticas revelam que $40 \%$ dos afastamentos funcionais são motivados por pequenas doenças e mal-estar, como gripes, dores nas costas, entorses e outras, as quais podem ser evitadas com medidas ergonômicas (SANTOS \& VAJDA, 2007). Além disto, pode-se observar o aumento dos agravos e ao surgimento de novo perfil de morbidade nos trabalhadores do país nas últimas décadas, em especial às Lesões por Esforços Repetitivos (LER) e os problemas de saúde mental decorrentes da reestruturação da economia e da produção, que sofre um momento de transição onde a mecanização e a especialização do trabalho ganharam espaço.

No Brasil, a dificuldade em se estabelecer um perfil dos trabalhadores e suas necessidades de atenção em saúde são concretas, tendo em vista, principalmente, que as características de produção são regionalizadas, onde cada microrregião econômica possui um perfil de atividade particular e seus trabalhadores possuem atividades diferentes. A diferença também existe no aparecimento de doenças ocupacionais relacionadas ao trabalho (DORT's) que estão consequentemente relacionadas ao tipo de atividade exercida (artesanato, indústria, agricultura, serviços, dentre outros).

A dificuldade de delinear um perfil e traçar formas de intervenção em saúde do trabalhador no âmbito nacional é um desafio a ser superado. Por tanto, analisar cada região e suas peculiaridades é a alternativa que este estudo propõe ao buscar conhecer de forma regionalizada a saúde do trabalhador, analisando indivíduos do setor de maior potencialidade na do estado do Pará, o setor industrial.

Levando em consideração a importância da vigilância para a manutenção da saúde, este trabalho tem como objetivo apresentar dados relativos aos problemas de saúde recorrentes nos trabalhadores do setor da indústria paraense, que integra a região amazônica, e ressaltar a importância de medidas com potencial de evitar agravos.

\section{METODOLOGIA}

Este estudo caracteriza-se por ser do tipo descritivo, de corte transversal com análise secundária de dados. Envolve 9.041 trabalhadores de 56 indústrias nacionais atuantes no Estado do Pará. O tamanho mínimo da amostra foi calculado considerando um erro amostral de $3 \%$, com intervalo de confiança de $95 \%$. 
As indústrias foram selecionadas de forma aleatória, sem que houvesse divisão proporcional de gênero, estado civil ou situação socioeconômica. Com isso, torna-se possível identificar o perfil dos trabalhadores do setor.

O levantamento de dados foi realizado a partir de documentos arquivados na gerência de qualidade de vida do Serviço Social da Indústria (SESI), no Estado do Pará. Os dados são oriundos de pesquisas de campo realizadas nas indústrias nos anos de 2013 e 2014, por profissionais capacitados que preencheram o instrumento da pesquisa, o questionário. $O$ questionário validado por Barros (1999) aplicou 89 perguntas objetivas direcionadas a saúde e ao estilo de vida de cada trabalhador.

O termo de consentimento fornecido pelo SESI/PA foi apresentado para apreciação e assinatura do entrevistado, especificando o caráter sigiloso das informações pessoais prestadas. Após essa etapa, foram utilizados os formulários completos, que continham a assinatura do trabalhador ou qualquer outro dado essencial para a precisão dos resultados, tornando a pesquisa ainda mais credível, descartando os arquivos que não correspondiam a este requisito.

O preenchimento do questionário foi feito pelos profissionais capacitados e pelo próprio funcionário da indústria pesquisada; respeitando a capacidade do indivíduo em preencher o seu próprio formulário no que diz respeito ao grau de escolaridade, nível de entendimento das perguntas propostas e problemas visuais.

Alguns itens do questionário foram obrigatoriamente preenchidos por profissionais da saúde, que fizeram avaliação antropométrica e aferição da pressão arterial (PA) nos trabalhadores, os mesmos observaram, no local de trabalho, a presença de doenças como obesidade e Hipertensão Arterial Sistêmica (HAS).

Após a coleta, os dados obtidos foram armazenados no banco de dados do Programa Microsoft Office Excel 2010 ${ }^{\mathrm{TM}}$ (Microsoft Corporation, Redmond, Estados Unidos). Destacase que a coleta de dados foi realizada pelo próprio SESI/PA e a utilização dos dados na análise passaram por aprovação do mesmo. A análise descritiva foi feita por meio de gráficos e tabelas.

O levantamento bibliográfico que serviu de suporte para esse estudo teve início em março de 2015 e foi acessado por diferentes bases de dados disponíveis on-line, tais como a Bireme, que engloba os sistemas Scielo, Lilacs, Scad e a Biblioteca Cochrane; o National Center for Biotechnology Information (NCBI), que envolve o Pubmed, o online books e outras bases virtuais; o portal CAPES e buscadores internacionais.

\section{RESULTADO}

A partir dos dados coletados, observou-se que os trabalhadores da indústria na região amazônica são predominantemente do gênero masculino, com renda até três salários mínimos, casados, que se consideram chefes de família, ganham até três salários mínimos e possuem casa própria (Tabela 1).

Tabela 1: Caracterização de gênero, estado civil, posição na família, renda mensal, e residência dos trabalhadores da indústria amazônica

\begin{tabular}{ll}
\hline \hline Variável & Percentual \\
\hline Gênero & \\
\hline Feminino & 19,41 \\
Masculino & 78,06 \\
\hline Estado civil e posição na família & \\
\hline Casados ou em união estável & 60,01
\end{tabular}


Declaram ser chefes de família 69,98

\begin{tabular}{ll}
\hline Renda mensal & \\
\hline Ganham até 3 SM & 76,69 \\
Ganham de 3 a 10 SM & 15,94 \\
Ganham mais do que 10 SM & 4,72 \\
Não sabem ou não responderam & 0,58 \\
\hline Residência & 68,79 \\
\hline Própria & 31,21 \\
\hline Alugada ou emprestada &
\end{tabular}

Legenda: SM: Salários Mínimos

Fonte: Autor, 2016.

A pesquisa, por ter ouvido e avaliado os trabalhadores da indústria amazônica, traz em seu escopo informações tanto de diagnóstico médico referido pelos trabalhadores quanto às informações coletadas por meio das avaliações clinicas de saúde. Os resultados desta investigação culminaram na eminencia de problemas metabólicos, vasculares, do aparelho locomotor e psicológicos.

Os problemas identificados através dos relatos dos trabalhadores revelam que, com relação a problemas vasculares, $6,57 \%$ dos trabalhadores das industrias amazônicas relataram ser hipertensos. $\mathrm{Na}$ avaliação clínica para a aferição da PA, $24,58 \%$ dos sujeitos apresentaram alteração nos valores de referência adotados pelo diagnóstico (normotenso até $140 / 90 \mathrm{~mm}$ $\mathrm{Hg}$ ) (CARDIOLOGIA, 2010). Dentre os avaliados, o que chama atenção é o percentual de trabalhadores que referem não possuir diagnóstico médico de HAS. 19,16\% dos que apresentaram pressão arterial alterada, negam possuírem HAS (Figura 1).

Figura 1: Dados clínicos e percepção de saúde dos trabalhadores da Indústria Amazônica para Hipertensão Arterial Sistêmica (HAS)

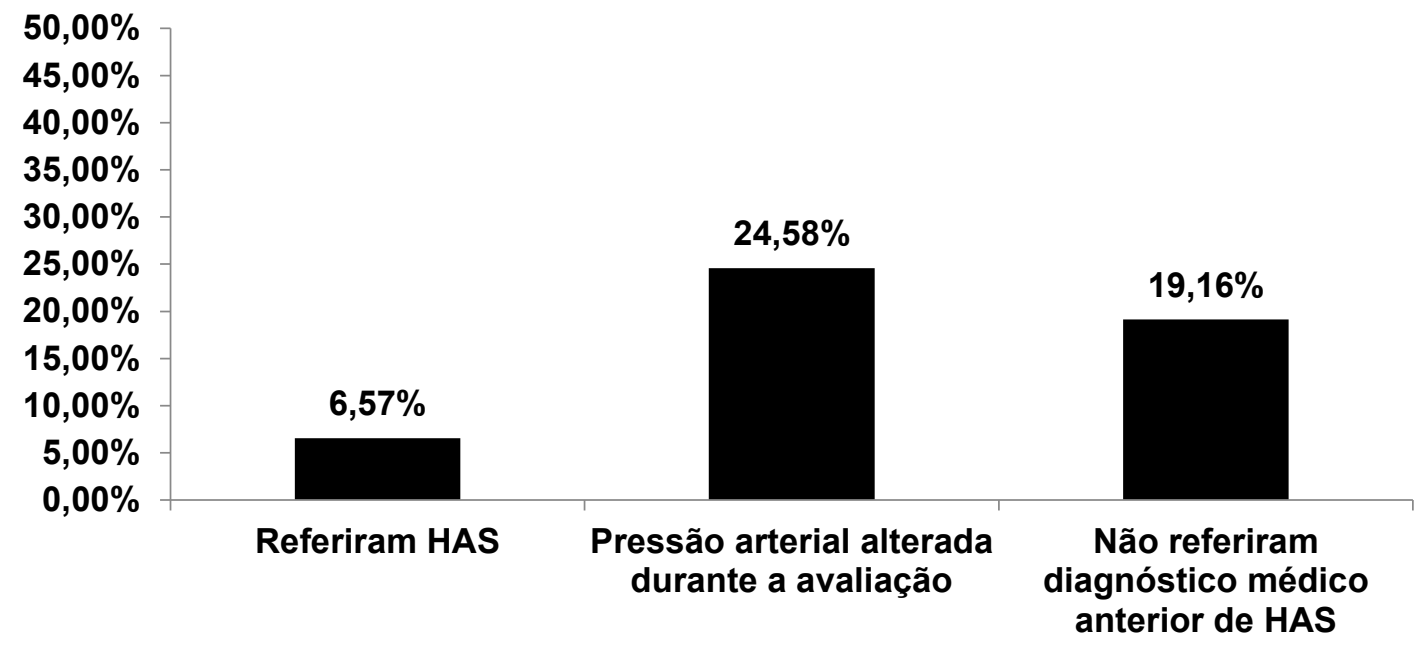

Fonte: Autor, 2016.

Os problemas do aparelho locomotor avaliados envolvem algumas das principais queixas encontradas nos consultórios de saúde ocupacional. Os problemas de coluna, tendinite e lesão por esforços repetitivos (LEER). Logo, os resultados indicam que $22,28 \%$ dos trabalhadores referiram problemas de coluna, e 4,50\% tendinite ou LEER (Figura 2). Já nas avaliações antropométricas, realizadas para identificar a obesidade, revelou-se que $14,66 \%$ 
dos trabalhadores possuíam algum grau de obesidade (Figura 2), variando entre obesidade grau 1 e grau 3 (obesidade mórbida).

Outro fator avaliado foi a ocorrência de diabetes na população industriaria. Para isso, a metodologia utilizada foi a entrevista. O relato do trabalhador já ajuda a dimensionar a gravidade do problema, visto que tal dado não foi confirmado por meio de exames clínicos. Por tanto, $2,12 \%$ dos trabalhadores relataram ter diabetes (Figura 2), sem especificar qual tipo (Diabetes tipo I ou II).

Figura 2: Problemas musculoesquléticos, diabetes referida e obesidade trabalhadores da Indústria Amazônica

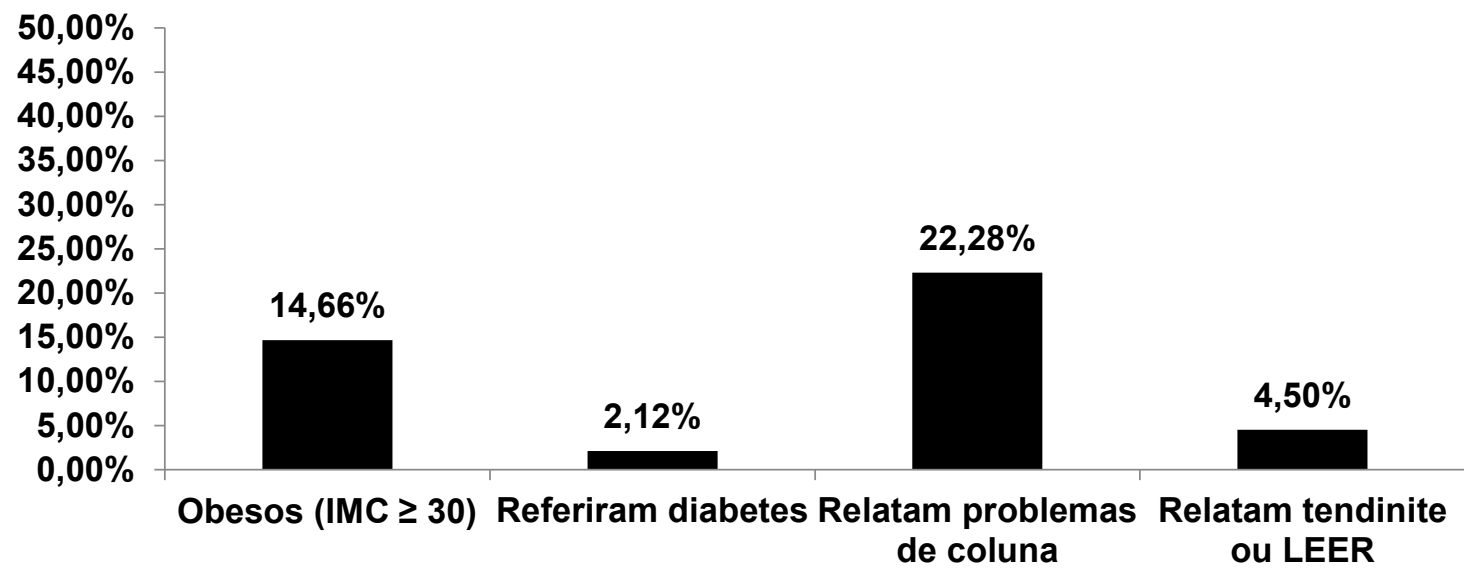

Fonte: Autor, 2016

Dentre os problemas de cunho psicológico, foi levantada a porcentagem de trabalhadores que relatam depressão, chegando a $2,82 \%$ dos avaliados (Figura 3 ). Contudo, outros dados foram levantados para posterior correlação. A porcentagem de entrevistados que apresentaram algum transtorno de depressão, identificados a partir de suas respostas ao questionário; e a porcentagem destes que não referem diagnóstico anterior de depressão, sendo respectivamente $9,11 \%$ e $8,52 \%$ dos entrevistados (Figura 3 ).

Figura 3: Dados clínicos e percepção de saúde dos trabalhadores da Indústria Amazônica para Depressão

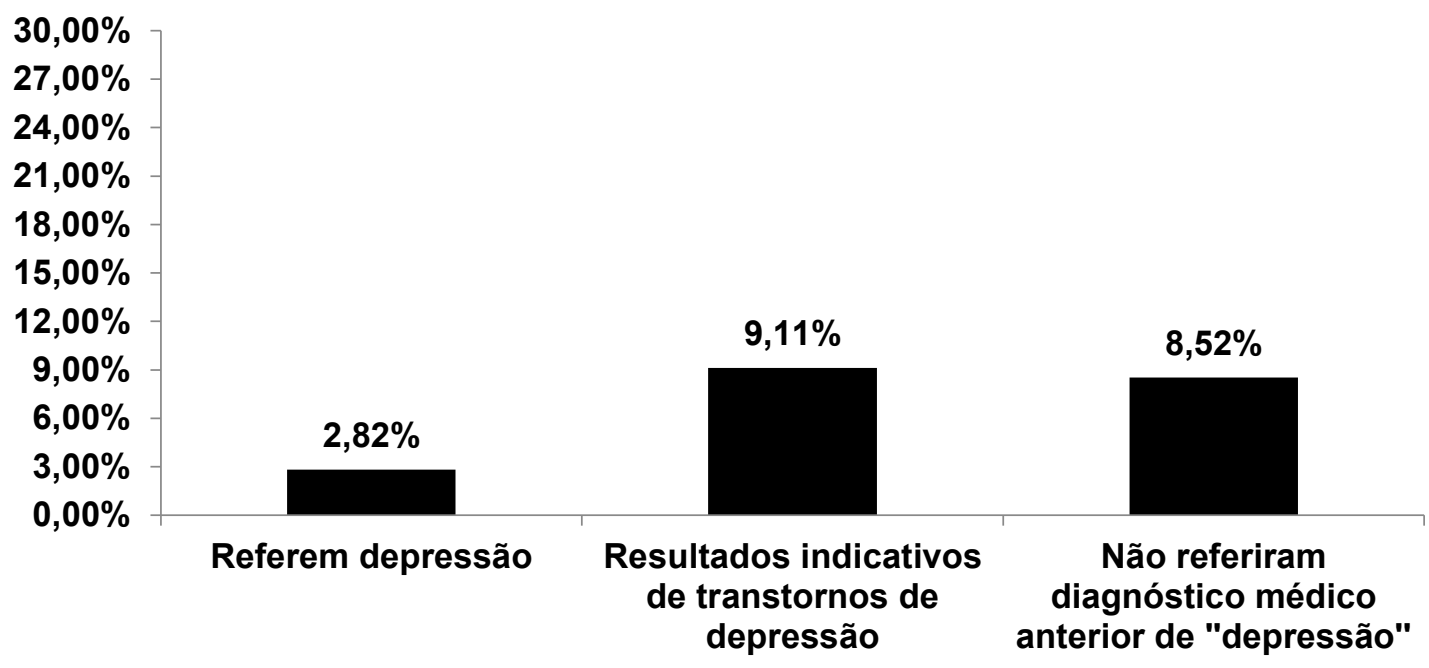

Fonte: Autor, 2015. 


\section{DISCUSSÃO}

O perfil do trabalhador da indústria amazônica se revela muito semelhante ao de outras regiões do país com relação às variáveis gênero, renda e estado civil (SILVA, 2011), onde se observa a prevalência de industriários do gênero masculino, com renda de até três salários mínimos e casados. Por tanto, a prevalência de homens e pessoas com baixa renda já era esperada como reflexo do tipo de atividade exercida, levando em consideração que muitas atividades industriais exigem força e resistência física, como as atividades extrativistas, agrícolas e construção civil.

Com relação aos dados de saúde, o presente estudo revela que porcentagem dos trabalhadores que relataram diagnóstico médico de diabetes $(2,12 \%)$ é menor quando comparada com a média nacional $(6,2 \%)$ apresentada pela última Pesquisa Nacional de Saúde (PNS) (IBGE, 2013). O surgimento desta estatística pode demonstra que as atividade exercidas pela indústria, que em muitos casos exigem força e resistência física, beneficiam a manutenção da saúde do trabalhador ao proporcionar uma rotina diária de atividade física.

Apesar da amostra ter sido predominantemente de homens (78,06\%), que comprovadamente possuem menor chance de desenvolver diabetes, ainda assim é possível afirmar que a influência de gênero não tem o potencial de influenciar de forma tão significativa aos resultados, já que as mulheres $(7,0 \%)$ e homens $(5,4 \%)$ apresentaram maior proporção de diabetes que a apresentada por esta pesquisa (IBGE, 2013).

A pesquisa ainda mostra que $14,06 \%$ estavam obesos, o que remete ao fato de que o desenvolvimento da obesidade perpassa pelos hábitos alimentares, com dieta rica em alimentos hipercalóricos e consumo baixo de alimentos saudáveis, como cereais e hortaliças.

De modo geral, com relação a alimentação, muitas indústrias possuem refeitório e disponibilizam alimentação baseada na ingestão de alimentos saudáveis. Porém, sem acompanhamento nutricional para o trabalhador no momento da eleição da dieta oferecida, as empresas não conseguem adequar hábitos positivos na alimentação de seus empregados (GERALDO, BANDONI \& JAIME; 2008). Assim, a ocorrência de doenças crônicas não transmissíveis pode aumentar consideravelmente, colocando em jogo a saúde do trabalhador.

Os locais de trabalho são um excelente espaço para oferecer programas de promoção à saúde, em particular aqueles voltados para a redução de fatores de risco para doenças metabólicas e cardiovasculares. A educação em saúde deve ser praticada no dia-dia das empresas. Pessoas com obesidade, diabetes e HAS (patologias associadas ao consumo excessivo de sal e açucares) demonstram preocupação em se educar para adquirir a capacidade de controlar possíveis agravos a saúde, promovendo um estilo de vida saudável, e é neste contexto que o setor de saúde da empresa deve agir. O ideal é que essa consciência venha antes da patologia instalar-se, principalmente porque a mudança de atitude não reflete somente no indivíduo em si, mas também em sua família e comunidade, pois a educação em saúde possui a característica de multiplicar para prevenir (TORRES et. al., 2011; FERREIRA et.al., 2006; SANTOS \& LIMA, 2008).

No levantamento para HAS, $24,58 \%$ apresentaram pressão arterial alterada no momento da avaliação, valor este que merece atenção a nível epidemiológico devido a relação da alimentação baseada em consumo excessivo de sal e o desenvolvimento de HAS, gerando assim mais um dado que alerta para a necessidade de programas de educação alimentar nas indústrias. (GERALDO, BANDONI \& JAIME; 2008).

Apesar de muitos trabalhadores terem apresentado a pressão arterial alterada durante a avaliação clinicas, somente $6,57 \%$ referiram diagnóstico de HAS. Por tanto, várias conclusões podem ser fomentadas com estes dados, principalmente porque, existe uma diferença significativa $(\alpha>0,5)$ entre o percentual das pessoas que relataram HAS e as 
pessoas que apresentaram alteração na pressão arterial durante a avaliação clínica $(6,57 \%$ e $24,58 \%$, respectivamente).

Além disso, a PNS apresenta que a proporção de indivíduos de 18 anos ou mais que referem diagnóstico de HAS, no Brasil, é de 21,4\%; número significativamente superior ao apresentado pelo presente estudo $(\alpha>0,5)$ (IBGE, 2013). Sendo assim, uma quantidade importante de trabalhadores pode apresentar HAS e não saber, fato que coloca em alerta a proteção à saúde destes colaboradores.

Os problemas de coluna e musculoesqueléticos relacionados ao trabalho merecem destaque quando se fala em proteção e promoção à saúde, já que alguns componentes tecnológicos e atividades da indústria são barreiras para a manutenção da plena saúde física. No presente estudo, a taxa de trabalhadores que relataram problemas de coluna $(22,28 \%)$ chama a atenção por ser maior que a média nacional $(13,50 \%)$, de acordo com a Pesquisa Nacional por Amostra de Domicílios (PnaD) (IBGE, 2008).

Algumas medidas podem ser incorporadas às empresas para que a ocorrência de problemas osteoarticulares não acometam seus empregados, principalmente para aqueles que realizam trabalhos manuais em setores administrativos, que sofrem grande risco de desenvolver problemas físicos devido ao trabalho estático (BIFF, 2006).

O incentivo à atividade física regular, controle da obesidade (fator predisponente a problemas de coluna) programas de ergonomia, ginástica laboral, educação em saúde, dentre outros tipos de atividades relatados na literatura, são aconselháveis por serem considerados como protetores e promotores da saúde física e mental (MONTEIRO, ALEXANDRE \& RODRIGUES, 2006; PADEZ, 2002).

Os locais de trabalho podem afetar o desempenho do trabalhador, levando a fadiga, desgaste e danos físicos. Por tanto, análise ergonômica como ferramenta para o controle de agravos a saúde é um caminho para a resolução de problemas complexos que exige o conhecimento das tarefas, da atividade desenvolvida para realizá-las e das dificuldades enfrentadas para se atingirem o desempenho e a produtividade exigidos (SLACK et al.,2002).

Dados com relação aos transtornos de cunho psicológico demonstraram que $9,11 \%$ dos trabalhadores da indústria amazônica tiveram resultados indicativos de depressão; $8,52 \%$ referiram diagnóstico médico anterior de depressão, e 2,82\% declaram possuir depressão. Estes itens merece uma investigação mais detalhada, já que os dados disponibilizados pelo SESI/PA não dá base para que se possa afirmar que os trabalhadores, que um dia já apresentaram diagnóstico médico de depressão, são os mesmos que apresentaram sinais indicativos de depressão durante a investigação. Apesar de não ser possível afirmar, é intuitivo que a maioria dos trabalhadores que apresentam resultados indicativos de depressão não sabem que possuem tal transtorno, já que a porcentagem de trabalhadores que relatam diagnóstico clínico $(2,82 \%)$ é menor que os que apresentaram o transtorno durante a investigação $(9,11 \%)$.

Quando indicadores de depressão e ansiedade evidenciam problemas psicológicos, o empregador já pode inferir que o problema pode está partindo do próprio trabalho. A saúde e as boas condições de trabalho oferecidas pela empresa resultam em melhor desempenho e maior satisfação do colaborador e da organização. Por isso, é dever do empregador identificar se a exigência de agilidade na execução de tarefas, o medo de perder o emprego, condutas de dominação para com o empregado, a competitividade entre os empregados, dentre outros, estão influenciando o desvio de comportamento, que pode levar a um leve comprometimento psíquico ou a uma psicopatia mais severa (FABRIN \& CASTRO, 2015).

A solução para problemas psicológicos precisa de uma análise dos casos, de forma particular, onde o trabalhador deve sempre ser avaliado junto a sua atividade laboral. Sendo assim, lidar com situações particulares requer a intervenção de profissionais qualificados para o diagnóstico e tratamento das afecções psicológicas. Esse fato é relevante à 
prevenção em saúde, já que a aplicação da intervenção deve ser elencada de acordo com a situação apresentada dentro da empresa, evitando assim, gastos desnecessários e execução de medidas ineficazes (FABRIN \& CASTRO, 2015).

\section{CONCLUSÃO}

Considerando que as atividades industriais dentro do território brasileiro se diferenciam e acompanham as características geoeconômicas de cada região, tomou-se por instrumento a análise de fatores de saúde dos trabalhadores de indústrias atuantes no Estado do Pará, que integra grande parte da região amazônica, e assim, foi possível identificar a prevalência de problemas de cunho físico e psicológico para esta população. Os resultados refletem indicadores positivos e negativos.

Pode-se destacar como indicadores positivos o menor número de pessoas que relatam diabetes e HAS quando comparados a população brasileira em geral. Já como indicadores negativos, os problemas de coluna, que potencialmente estão relacionados a fatores ambientais e ergonômicos, e que apresentam grande incidência na população industriaria da do Estado do Pará. Além disso, a depressão também aparece como um indicador negativo, visto que a maioria dos trabalhadores que apresentam resultados indicativos de depressão não sabem que possuem tal transtorno, devido à porcentagem de trabalhadores que relatam diagnostico de depressão ser menor que a porcentagem dos que apresentaram sinais indicativos de depressão durante a investigação.

Com base em toda essa fundamentação, pesquisa e análise, conclui-se que a vigilância à saúde do trabalhador da indústria amazônica merece uma atenção mais intensa, tanto com relação às políticas públicas quanto às medidas de caráter privado dos empresários, com vistas para o crescimento social e econômico local e o bom andamento das atividades industriais da região que, além de tudo, também é carente de pesquisas voltadas à ergonomia e saúde do trabalhador.

Devido à grande diversidade de culturas e etnias no Brasil, seria interessante a realização de estudos em outros locais do país para que se possa entender a realidade de cada região, correlacionando as características locais. A realização do mesmo estudo para o biênio de 2015/2016 terá grande relevância cientifica, uma vez que será possível identificar e comparar a evolução das condições de saúde dos industriários; que supostamente trarão reflexo positivo ao revelarem quais pontos merecem maior atenção para a promoção à saúde dos trabalhadores.

\section{REFERÊNCIAS BIBLIOGRÁFICAS}

DE BARROS, M.V.G. Atividades físicas no lazer e outros comportamentos relacionados à saúde dos trabalhadores da indústria no Estado de Santa Catarina, Brasil. Florianópolis: UFSC, 1999, 64f. Dissertação (Mestrado em Educação física) - Centro de Desportos, Universidade Federal de Santa Catarina, Florianópolis, 1999.

BRASIL. Decreto-lei $n^{\circ} 7.602 / 2011$, de 07 de novembro de 2011. Dispõe sobre a Política Nacional de Segurança e Saúde no Trabalho. Diário Oficial [da] República Federativa do Brasil, Poder Executivo, Brasília, DF, 08 de nov. 2011. Disponível em: <http://www.planalto.gov.br/ccivil_03/_Ato2011-014/2011/Decreto/D7602.htm>. Acesso em: 05 mar. 2016.

BRASIL. Ministério da Saúde. Portaria $n^{\circ}$ 1.823, de 23 de agosto de 2012. Diário Oficial [da] República Federativa do Brasil, Poder Executivo, Brasília, DF, 24 de ago. 2012. n 165, Seção I, p. 46-51. Disponível em: <http://bvsms.saude.gov.br/bvs/saudelegis/gm/2012/prt 1823_23_08_2012.html>. Acesso em: 12 mar. 2016. 
SOCIEDADE BRASILEIRA DE CARDIOLOGIA. VI Diretrizes Brasileiras de Hipertensão. Disponível em: <http://www.anad.org.br/profissionais/images/VI_Diretrizes_Bras_ Hipertens _RDHA_648> 5.pdf, Acesso em: 19 mar. 2016.

COUTO, H.A. Ergonomia aplicada ao trabalho: manual técnico da máquina humana. Belo Horizonte: ERGO Editora, 1995. v. 1, 353 p.

INSTITUTO BRASILEIRO DE GEOGRAFIA E ESTATíSTICA (IBGE). Pesquisa Nacional de Saúde Percepção do Estado de Saúde, Estilos de Vida e Doenças Crônicas. Rio de Janeiro: IBGE, 2013. 181p.

INSTITUTO BRASILEIRO DE GEOGRAFIA E ESTATÍSTICA (IBGE). Pesquisa Nacional por Amostra de Domicílios Pnad - 2008. Rio de Janeiro: IBGE, 2008. 245p.

FABRIN, J.F.; CASTRO, M. As possíveis influências do ambiente de trabalho no adoecimento psíquico de trabalhadores ativos do município de Seara-SC. Tecnológica, v. 2, n. 1, p. 88-108, 2015.

FERREIRA, S.R.G. et al. Frequência de hipertensão arterial e fatores associados: Brasil, 2006. Rev Saúde Publica, São paulo, n. 43 supl. 2, p. 98-106, 2009.

GERALDO, A.P.G.; BANDONI, D.H.; JAIME, P.C. Aspectos dietéticos das refeições oferecidas por empresas participantes do Programa de Alimentação do Trabalhador na Cidade de São Paulo, Brasil. Rev Panam Salud Publica, São Paulo, n. 23, v. 1, p. 19-25, 2008.

MARQUES, A. et al. A Ergonomia como um Fator Determinante no Bom Andamento da Produção: um Estudo de Caso. Revista Anagrama, São Paulo, ano 4, 1. Ed., p. 14, 2010.

MONTEIRO, M.S.; ALEXANDRE, N.M.G.; RODRIGUES, C.M. Doenças músculo-esqueléticas, trabalho-esqueléticas, trabalho e estilo de vida entre trabalhadores de estilo de uma instituição pública de saúde. Rev. esc. enferm. USP, São Paulo, v.1, p. 20-25, 2006. Disponível em: <http://www.scielo.br/pdf/reeusp/v40n1/a02v40n1.pdf>Acesso em: 13 Fev. 2016.

PADEZ, C. Actividade física, obesidade e saúde: uma perspectiva evolutiva. Revista Portuguesa de Saúde Pública, Lisboa, n. 20, v. 1, p. 11-19, 2002.

SANTOS, Z.M.S.A.; LIMA, H.P. Tecnologia educativa em saúde na prevenção da hipertensão arterial em trabalhadores: análise das mudanças no estilo de vida. Texto Contexto-enferm., Florianópolis, $\mathrm{n}$. 17 , v. 1, p. 90-97, 2008.

SILVA, S.G. et al. Fatores associados à inatividade física no lazer e principais barreiras na percepção de trabalhadores da indústria do Sul do Brasil. Cad. Saúde Pública, Rio de Janeiro, v. 27, n. 2, p. 249-259, 2011. Disponível em: <http://www.scielosp.org/pdf/csp/v27n2/06.pdf> Acesso em: 22 Jan. 2016.

SLACK, N,; CHAMBERS, S.; JOHNSTON, R. Administração da Produção. Tradução de Maria Teresa Corrêa de Oliveira, Fábio Alher. 2. ed., São Paulo: Atlas, 2002. p. 29-62.

TOLDRA, R.C. et al. Facilitadores e barreiras para o retorno ao trabalho: a experiência de trabalhadores atendidos em um centro de referência em saúde do trabalhador - SP, Brasil. Rev. bras. saúde ocup., São Paulo, v. 35, n. 121, p. 10-22, 2010. Disponível em: <http://www.scielo.br/scielo.php?script=sci arttext\&pid=S0303-76572010000100003\&lng= en\&nrm=iso>. Acesso em: 10 Jan. 2016.

TORRES, H.C. et al. Intervenção educativa para o autocuidado de indivíduos com diabetes mellitus. Acta paul. enferm., São Paulo, v. 24, n .4, p. 514-519. Disponível em: http://www.scielo.br/pdf/ape/v24n4/a11v24n4.pdf>. Acesso em: 13 Jan. 2016. 